\title{
Lugano Lymphoma Response Classification No Response/Stable Disease by $\mathrm{CT}$
}

National Cancer Institute

\section{Source}

National Cancer Institute. Lugano Lymphoma Response Classification No

Response/Stable Disease by CT. NCI Thesaurus. Code C142793.

Less than 50 percent decrease from baseline in the sum of the product of the perpendicular diameters of up to 6 dominant, measurable nodes and extranodal sites; no criteria for progressive disease are met. 